\title{
Prospects and Clinical Perspectives of Total-Body PET Imaging Using Plastic Scintillators
}

Paweł Moskal, PhD*, Ewa Ł. Stępień, PhD*

\section{KEYWORDS}

- J-PET • PET imaging • Total-body PET imaging • Multitracer imaging • Positronium imaging

- ${ }^{82} \mathrm{Rb}$-chloride $\bullet{ }^{44} \mathrm{Sc}-\mathrm{PSMA} \bullet$ Plastic scintillators

\section{KEY POINTS}

- Total-body PET opens a new diagnostic paradigm with prospects for personalized disease treatment, yet the high cost of the current crystal-based PET technology limits dissemination of totalbody PET in hospitals and even in the research clinics.

- The J-PET tomography system is based on axially arranged low-cost plastic scintillator strips. It constitutes a realistic cost-effective solution of a total-body PET for broad clinical applications.

- High sensitivity of total-body J-PET and triggerless data acquisition enable multiphoton imaging, opening possibilities for multitracer and positronium imaging, thus promising quantitative enhancement of specificity in cancer and inflammatory diseases assessment.

- An example of dual tracer analysis, becoming possible with total-body J-PET system, could be a concurrent application of Food and Drug Administration-approved ${ }^{82} \mathrm{Rb}-C h l o r i d e$ and $\left[{ }^{18} \mathrm{~F}\right] \mathrm{FDG}$, allowing simultaneous assessment of myocardium metabolic rate and perfusion of the cardiovascular system.

\section{INTRODUCTION}

As modern medicine advances toward personalized treatment of patients, it requires highly specific and sensitive tests to diagnose disease. The advent of total-body PET (TB-PET) systems ${ }^{1-6}$ opens new perspectives for the precision medicine enabling detection of pathologies on a molecular level in the whole patient body simultaneously, before they lead to the functional or structural abnormalities. ${ }^{7,8}$ TB-PET imaging creates a new paradigm for precision medicine, yet not fully exploited, enabling simultaneous imaging of metabolism rate in close and distant organs, reducing drastically the whole-body scan time or the dose delivered by the radiopharmaceutical agent (by a factor of approximately 40 with respect to typical current clinical PET systems). ${ }^{3}$ Introduction of
TB-PETs extends the PET application to diagnosis of a wider group of patients (eg, children and pregnant women) and systemic diseases, as for example, autoimmune diseases, cardiovascular diseases, or active rheumatoid diseases ${ }^{8-10}$ by simultaneous multiorgan kinetic modeling, currently not possible with the $\sim 25 \mathrm{~cm}$ axial filed-of-view scanners. ${ }^{11,12}$ Kinetic model-based parametric imaging may also deliver additional complementary diagnostic information to that available from standard static standardized uptake value (SUV) images. ${ }^{13-15}$

However, introduction of the TB-PET scanners in the common clinical practice encounters challenges of the high cost, estimated to approximately $\$ 10$ million or more for a single TB-PET system built based on the current technology

Marian Smoluchowski Institute of Physics, Jagiellonian University, ul. S. Lojasiewicza 11, Kraków 30-348, Poland

* Corresponding authors.

E-mail addresses: p.moskal@uj.edu.pl (P.M.); e.stepien@uj.edu.pl (E.Ł.S.) 
using crystal scintillators. ${ }^{4}$ High price is a serious barrier factor in using this device not only in hospital facilities but even in medical research clinics.

Therefore, there is a need for new technical solution that would enable to substantially decrease the cost of the extended axial field-of-view (AFOV) PET, where approximately half of the cost is in scintillators, and the other half of the costs is in silicon photomultipliers (SiPM) and electronics. ${ }^{16}$

The ongoing investigations aiming at cost reduction focuses on the possibilities of (1) scintillator thickness reduction ${ }^{17}$; (2) sparse detector configurations $^{18,19}$; and (3) application of BGO crystals ${ }^{20,21}$ with Cherenkov light readout for the improved timing properties. ${ }^{22-25}$

The reduction of detector thickness decreases the sensitivity approximately as a square of the reduction thickness coefficient, whereas the cost of scintillators decreases only linearly without reducing the costs of electronics. Similarly, in the sparse configuration, the sensitivity drops as square of the decrease of the number of detector components. ${ }^{18}$ An application of BGO crystals may reduce costs of the scintillators by a factor 2 to $3^{16}$; however, at the same time the costs may increase due to the increased requirements and complications for the of readout electronics needed for taking advantage of both scintillation and Cherenkov light.

Therefore, it is claimed that only a spectacular decrease in the price, while maintaining the key parameters such as total-body dynamic/kinetic imaging, will accelerate the dissemination process of the TB-PET imaging. ${ }^{26}$ In general it is also important to decrease the costs of the whole diagnostic process, which includes not only PET imaging enabling detection of the presence of diseased tissues based on SUV, but also histologic assessments of tissues requiring biopsies for distinguishing among the inflammatory, infected, and cancerous tissues and for evaluating the grade of cancer malignancy.

Therefore, the effective translation of TB-PET for wide use in clinics, in view of the precision diagnosis tailored to the individual patient, requires significant decrease of costs of the TB-PET construction with respect to the pioneering EXPLORER PET technology and an increase of specificity in cancer and inflammatory diseases assessment.

In this article, we discuss the principle of operation of the new Jagiellonian-PET (J-PET) tomography system developed at the Jagiellonian University, Cracow, Poland, based on axially arranged low-cost plastic scintillator strips, as contrasted with the current PET scanners with radially arranged expensive crystal blocks. J-PET enables cost-effective construction of PET with long AFOV, up to $2.5 \mathrm{~m}$ and more, which would enable high sensitivity imaging of the whole human body, with high and uniform sensitivity over the whole patient from the brain to the feet. Imaging with such a large AFOV would open new perspective for diagnosis of diseases affecting the body and brain simultaneously. J-PET may be constructed as a modular, light and portable TBPET, enabling reconfiguration of the tomographic volume, which may help to extend PET diagnosis further by including patients who cannot be examined with the standard PET due to factors such as obesity or claustrophobia.

Further on, we discuss the properties of the economic total-body J-PET (TB-J-PET), and its prospects for performing both the simultaneous multitracer imaging and positronium imaging. The newly proposed positronium imaging is being developed based on properties of positronium atoms produced in the intramolecular voids in the patient's cells during the routine PET diagnosis, ${ }^{27-30}$ and it is promising for enhancing the specificity of cancer and inflammatory diseases assessment in TB-PET diagnostics.

\section{PROSPECTS FOR TOTAL-BODY PET IMAGING WITH PLASTIC SCINTILLATORS}

The J-PET tomography scanner is constructed from plastic scintillator strips read out at both ends by matrices of SiPM. Schematic view of the axial cross-section of the scanner is shown in Fig. 1, whereas the left panel of Fig. 2 presents a perspective view. The position and time of the interaction of annihilation photons is determined by measurement of the times of scintillation light signals arrivals to the edges of the scintillators. ${ }^{31}$ Light attenuation by plastic scintillators is by more than 1 order of magnitude lower than in the crystal scintillators, and that enables effective light transport even in strips of a few meters' length (for example, attenuation length for BC408 plastic scintillator amounts to $380 \mathrm{~cm}$ compared with approximately $21 \mathrm{~cm}$ to $40 \mathrm{~cm}$ for the LYSO crystal). ${ }^{32-34}$ The signals from SiPMs are probed in the voltage domain with the time accuracy of approximately 20 ps by newly developed

aThe Netherlands has the tallest people in the world, where the average height of men is $1.83 \mathrm{~m}$ (https://adc. bmj.com/content/90/8/807). 

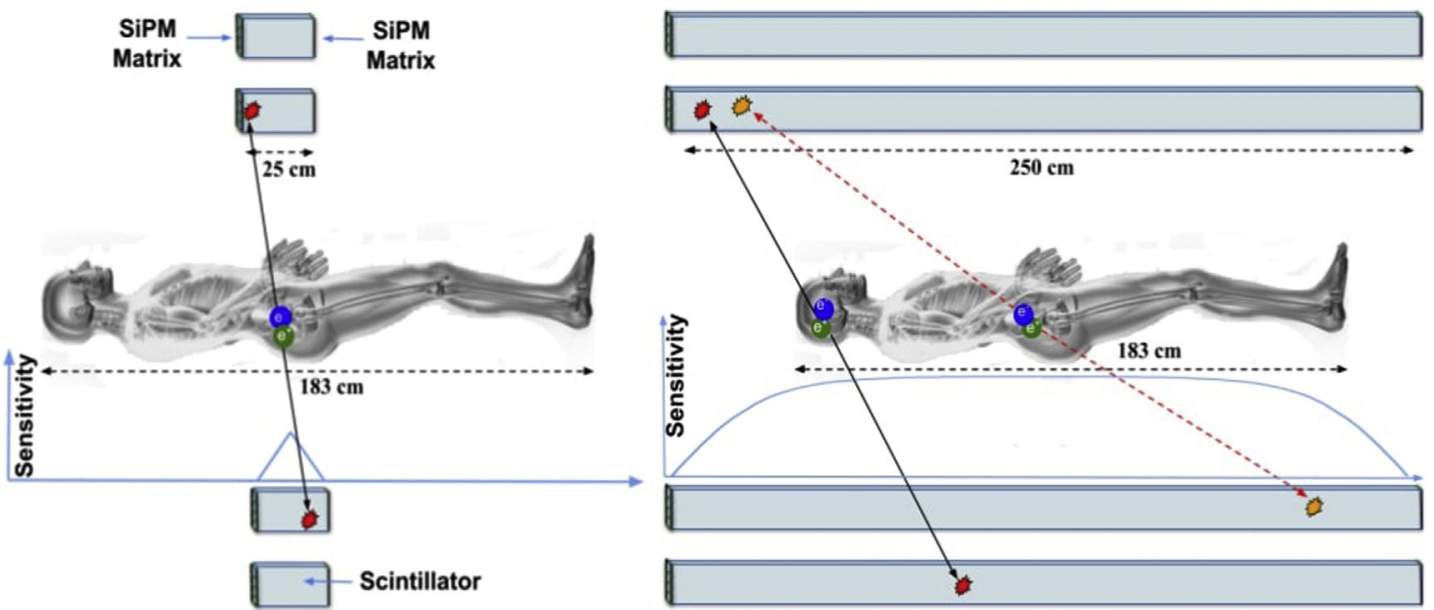

Fig. 1. Schematic view of the axial cross section of the J-PET tomograph composed of the 2 detection layers. Left and right panels illustrate the cases of J-PET with $25 \mathrm{~cm}$ and $250 \mathrm{~cm}$ AFOV and the 183-cm-tall patient. ${ }^{\text {a }}$ The single $^{-}$ detection module consists of a scintillator strip read out by two SiPM matrices. The cross section of the plastics strips designed for the TB-J-PET is $6 \mathrm{~mm} \times 30 \mathrm{~mm}$. Here it is presented not to scale. Solid and dashed arrows indicate exemplary lines of response (LOR) originating from $\mathrm{e}^{+} \mathrm{e}^{-}$annihilation. The interaction distance from the center of the scintillator is determined by the difference in times the two signals reach both ends of the scintillator strip. The position along the LOR is determined from time difference measured between two strips. In practice, more advanced methods of hit-time and hit-position were developed, which take advantage of the variation of the signal shape as a function of the hit-position. ${ }^{39,40}$ Superimposed charts indicate the sensitivity (in arbitrary units) along the AFOV. It was calculated using the formula described in reference. ${ }^{29}$ The presented sensitivity includes also the attenuation of annihilation photons in the patient. The attenuation in the patient flattens the sensitivity inside the long AFOV scanner. The value of sensitivity is given in arbitrary units, however the values in the left and right figures are shown in the same scale.
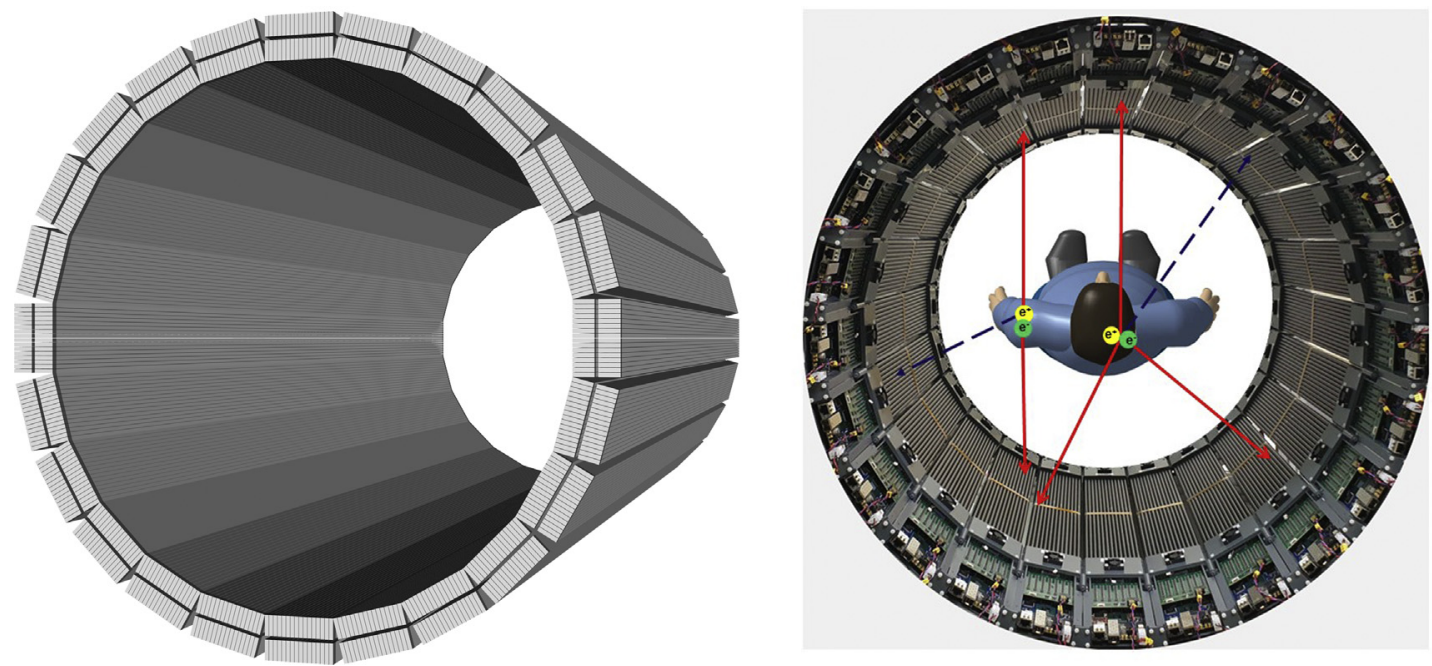

Fig. 2. (Left) Perspective view of the design of two-layer TB-J-PET scanner. Each layer consists of 24 modules each including 16 scintillator strips with cross-section of $6 \mathrm{~mm} \times 30 \mathrm{~mm}$. (Right) Photograph of the single-layer (with $50 \mathrm{~cm}$ AFOV) modular J-PET prototype with superimposed representations of electron-positron annihilation in the patient's body for 2 and 3 photon events (red solid arrows) and the associated prompt gamma rays (blue dashed arrows) emitted by the $\beta^{+} \gamma$ radionuclide such as for example, ${ }^{44} \mathrm{Sc}$ or ${ }^{82} \mathrm{Rb}$. The single-layer J-PET prototype with AFOV $=50 \mathrm{~cm}$ weighs only approximately $60 \mathrm{~kg}$. It consists of 24 modules each built from plastic scintillator strips (black) read out at both ends by SiPMs equipped with the dedicated front-end and digitizing electronics visible in the foreground. ${ }^{35,36}$ 
electronics ${ }^{35}$ and the data are collected by the novel triggerless and reconfigurable data acquisition system. ${ }^{36}$ The readout data are streamed to the Central Controller Module and then to a permanent storage. ${ }^{36}$ For data processing and simulations, a dedicated software framework was developed. ${ }^{37}$ The hit-position and hit-time are reconstructed by the dedicated reconstruction methods based on the compressing sensing theory $^{38}$ and the library of synchronized model signals. ${ }^{39}$

\section{Sensitivity Gain}

Plastic scintillators $\left(1.02-1.06 \mathrm{~g} / \mathrm{cm}^{3}\right)^{32,41}$ are approximately 7 times less dense than LYSO crystals $\left(7.0-7.4 \mathrm{~g} / \mathrm{cm}^{3}\right)^{42}$ and hence less effective for the registration of annihilation photons. However, the axial arrangement of plastic strips with the SiPM readout at the ends provides compensation for the lower registration efficiency by application of multilayer geometry with concentric detector layers, ${ }^{43}$ as is visualized in Figs. 1 and 2 (left). The overall imaging sensitivity depends on the geometric acceptance of the detector, efficiency of the registration of annihilation photons, efficiency of the selection of events contributing to the PET image formation, and on the attenuation of photons in the imaged object/patient. Annihilation photons emitted at small angles to the cylinder axis (dashed LOR in Fig. 1, right) are strongly attenuated in the patient body and effectively the sensitivity for registration and selection of image forming events saturates at approximately $50 \mathrm{~cm}$ from the tomograph edges, as indicated by the blue line in the right panel of Fig. 1, instead of growing almost linearly toward the detector center as is in the case of short AFOV systems, as indicated in the left panel of Fig. 1. The solid and dashed lines in Fig. 1 indicate examples of a moderately and very oblique lines-of-response, respectively.

To quantitatively estimate the gain in the scanner sensitivity with the extension of the AFOV and to compare the sensitivity gains between the crystal and plastic based scanners, we performed calculations under the assumption that the compared scanners are in the form of a cylinder with the diameter of $80 \mathrm{~cm}$ and thickness of $1.81 \mathrm{~cm}$ (LYSO) and $6 \mathrm{~cm}$ (2 layers of plastic scintillators with $3-\mathrm{cm}$ thickness each). In the left panel of Fig. 3 we present obtained results taking into account attenuation of photons in the body

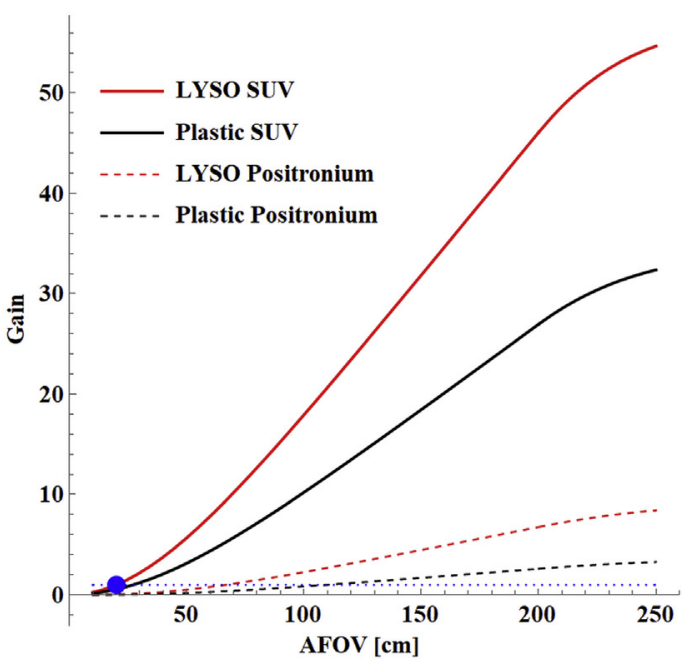

Fig. 3. Sensitivity gain with respect to the $20 \mathrm{~cm}$ AFOV LYSO PET (indicated with blue) for a small lesion ( $L=1 \mathrm{~cm}$ object), single organ ( $L=10 \mathrm{~cm}$ object), brain with torso $(\mathrm{L}=100 \mathrm{~cm}$ long object) and the total body ( $200 \mathrm{~cm}$ long object). Blue dotted line shows gain equal to unity. Sensitivity includes the probability of registration and selection of image forming events as well as attenuation of photons in the body (water phantom with $20 \mathrm{~cm}$ diameter and $200 \mathrm{~cm}$ long). Results as a function of the axial length for LYSO (1.81 cm thick) and plastic (two 3-cm-thick layers) PET detectors are shown. The meaning of line types is explained in the legends. The red and black colors indicate results for the LYSO and plastic scintillators, respectively. (Left) Results of sensitivity gain for the standard $2 \gamma$ annihilation photons imaging. (Right) The sensitivity for the standard $2 \gamma$ imaging of the SUV is compared to the sensitivity of the triple-coincidence $2 \gamma+$ prompt positronium imaging. ${ }^{27-29}$ In the right panel only the results obtained for the total-body imaging (for source of $L=200 \mathrm{~cm}$ ) are shown. Calculations were performed assuming energy of the prompt gamma of $1157 \mathrm{keV}$ as emitted by the ${ }^{44} \mathrm{Sc}$ isotope. ${ }^{44}$ 
as well as the changes of interaction probability as a function of the angle under which the photon is entering the detector. The attenuation was estimated approximating the body with a water phantom of $20 \mathrm{~cm}$ diameter and $200 \mathrm{~cm}$ long.

For the whole-body scan (estimated for the 200-cm-long object) the sensitivity for the $200 \mathrm{~cm}$ AFOV scanner, with respect to the $20 \mathrm{~cm}$ AFOV PET, is enhanced by a factor of approximately 46 for the LYSO based and by a factor of approximately 27 for the plastic based TB-J-PET scanner, respectively. The gain for brain plus torso imaging (approximated by a $100-\mathrm{cm}$-long object) saturates for the AFOV of approximately $150 \mathrm{~cm}$, and increases by a factor of approximately 28 for the LYSO PET and by a factor of 15 for the plastic PET. In case of the single organ or a lesion (short $10 \mathrm{~cm}$ or $1 \mathrm{~cm}$ objects) the gain is already saturated for systems with $70 \mathrm{~cm}$ AFOV and reaches values of approximately 3.8 and 2.3 (for the 10-cm object) and 3.0 and 1.8 (for the $1 \mathrm{~cm}$ object) for LYSO and plastic PET, respectively.

\section{National Electrical Manufacturers Association Characteristics: Spatial Resolution, Noise Equivalent Count Rate, Sensitivity and Scatter Fraction}

The spatial resolution, sensitivity, scatter fraction and noise equivalent count rate (NECR) for the J-PET were estimated ${ }^{45}$ using GATE simulation software, ${ }^{46}$ following the National Electrical Manufacturers Association (NEMA) NU 2 to 2012 standards.

For a strip length of $100 \mathrm{~cm}$ with cross-section of $4 \mathrm{~mm} \times 20 \mathrm{~mm}$ and an additional layer of wavelength shifter as the readout, ${ }^{47}$ the point spread function (PSF) in the center of the scanner is equal to $3 \mathrm{~mm}$ (radial, tangential) and $6 \mathrm{~mm}$ (axial). For the double layer geometry, the NECR peak of $300 \mathrm{kcps}$ is reached at $40 \mathrm{kBq} / \mathrm{mL}$ activity concentration and the sensitivity at the center amounts to $14.9 \mathrm{cps} / \mathrm{kBq}$. Whereas for the TB-J-PET with AFOV of $200 \mathrm{~cm}$ and a scintillators with crosssection of $6 \mathrm{~mm} \times 30 \mathrm{~mm}$, the PSF is estimated to $4.9 \mathrm{~mm}$ (radial, tangential) and $7 \mathrm{~mm}$ (axial), the NECR peak of $600 \mathrm{kcps}$ is reached at 25 $\mathrm{kBq} / \mathrm{mL}$ activity concentration, and the sensitivity at the center amounts to $38 \mathrm{cps} / \mathrm{kBq}$. The scatter fraction is estimated to approximately $35 \%$. These values can be compared with PSF of $4 \mathrm{~mm}$ and $3 \mathrm{~mm}$, the NECR of $1435 \mathrm{kcps}$ and $1000 \mathrm{kcps}$, sensitivity at the center of $191.5 \mathrm{cps} / \mathrm{kBq}$ and $55 \mathrm{cps} / \mathrm{kBq}$ and scatter fraction of $35.8 \%$ and $32 \%$ for UEXPLORER ${ }^{16}$ and Penn PET EXPLORER, ${ }^{2}$ respectively.

\section{Multiphoton Imaging}

High sensitivity of TB-PET scanners opens opportunities for application in PET scans of events with the emission of 3 or more photons. The triggerless data acquisition system of the J-PET tomograph ${ }^{36}$ enables detection of all events including multiphoton annihilations and prompt gammas, not restricted to the standard double annihilation photons coincidences as it is in the current PET scanners. Triggerless mode opens the possibility for flexible selection of events at the software level. ${ }^{37}$ This may include selection of double, triple and in general multicoincidence events. In particular it enables registration and identification of $2\left(\mathrm{e}^{+} \mathrm{e}^{-}\right.$ $\rightarrow 2 \gamma$ ) and 3 photon $\left(\mathrm{e}^{+} \mathrm{e}^{-} \rightarrow 3 \gamma\right.$ ) annihilations, as well as prompt gamma emitted in case of some isotopes referred to as $\beta^{+} \gamma$ emitters, such as for example, ${ }^{10} \mathrm{C},{ }^{14} \mathrm{O},{ }^{22} \mathrm{Na},{ }^{34} \mathrm{Cl},{ }^{44} \mathrm{Sc},{ }^{48} \mathrm{~V}$, ${ }^{52} \mathrm{Mn},{ }^{55} \mathrm{Co},{ }^{60} \mathrm{Cu},{ }^{66} \mathrm{Ga},{ }^{69} \mathrm{Ge},{ }^{72} \mathrm{As},{ }^{76} \mathrm{Br},{ }^{82} \mathrm{Rb}$, ${ }^{86} \mathrm{Y},{ }^{94} \mathrm{Tc},{ }^{110} \mathrm{In},{ }^{124} \mathrm{I} .{ }^{44}$ Some examples of multiphoton events are shown pictorially in the right panel of Fig. 2. After emission of positron the $\beta^{+} \gamma$ emitters change into the daughter nucleus in an excited state. The daughter nucleus subsequently de-excites through emission of 1 or several gamma quanta. For example, in the case of Scandium the reaction chain is as follows: ${ }^{44} \mathrm{Sc} \rightarrow{ }^{44} \mathrm{Ca}^{*} \mathrm{e}^{+} v \rightarrow{ }^{44} \mathrm{Ca} \gamma \mathrm{e}^{+} v$, where $v$ denotes neutrino that escapes from the body undetected.

In the current PET imaging procedures, prompt gammas and $\mathrm{e}^{+} \mathrm{e}^{-} \rightarrow 3 \gamma$ annihilations constitute a source of unwanted background. In the body a fraction of $3 \gamma$ annihilation events constitutes approximately $0.5 \%$ only ${ }^{28}$; however, prompt gamma may accompany almost each annihilation as it is in the case of for example, ${ }^{44} \mathrm{Sc}$ radionuclide. ${ }^{44}$ However, these multiphoton coincidences may be useful for diagnosis. ${ }^{28}$ Capability of J-PET to register and identify the signals from prompt gammas and from $2 \gamma$ and $3 \gamma$ annihilations allows for tagging the events originating from various isotopes. This information enables classification of registered events according to the radiotracer, and hence enables diagnosis with 2 or more tracers simultaneously during single PET examination. ${ }^{48}$ Therefore, in case of the $\beta^{+} \gamma$ emitters, an additional prompt gamma may be used not only for improving the spatial resolution by combining $2 \gamma$ PET with the various types of Compton cameras for the registration of the prompt gamma as discussed, for example, in Refs. ${ }^{49-53}$, but it may also be used for the simultaneous multitracer imaging $^{48}$ and for the newly developed positronium lifetime imaging, which is a promising approach for the in vivo assessment of tissue pathology. ${ }^{27-29}$ 


\section{Example of Multitracer Imaging Applications with Total-Body Jagiellonian-PET}

Despite potential advantage in PET imaging, especially in TB-PET, and broad accessibility of suitable tracers, the $\beta$-emitters application is still limited, and to date only a few clinical trials have been conducted. The summary of published results of the preclinical and clinical studies is presented in Table 1. Among the previously listed 18 isotopes, only one is approved by the Food and Drug Administration (FDA) $\left({ }^{82} \mathrm{Rb}\right)$ and only 2 $\left({ }^{44} \mathrm{Sc}\right.$ and $\left.{ }^{124} \mathrm{I}\right)$ are clinically applied and few of them were tested preclinically. ${ }^{54}$

The trivalent $\beta$-emitters as ${ }^{44} \mathrm{Sc},{ }^{66} \mathrm{Ga}$, and monovalent ${ }^{124} I$ are especially promising because they

i. Can be delivered by the same class of chelators or carries as their commonly used analogues (isotopes): DOTA, ${ }^{61} \mathrm{IMP},{ }^{58} \mathrm{PIB},{ }^{71}$ NOTA, ${ }^{62}$ PSMA-617 67 ii. Can be attached to tracers approved in clinical trials: PSMA-617, ${ }^{68,74}$ Herceptin (trastuzumab), ${ }^{70}$ cetuximab ${ }^{75}$

iii. Can recognize the same targets: EGFR ${ }^{58}$ HER2 ${ }^{44}$ Sc-DOTA-ZHER2:342, ${ }^{72-}$ CAIX, ${ }^{55,56}$ Integrin $\alpha_{\mathrm{V}} \beta_{3}{ }^{59}$ PSMA, ${ }^{69}{\text { SST } 2 R^{64}}^{64}$

iv. Can be used in dual analysis with 2 radiotracers "pairs": ${ }^{124}$ I-trastuzumab/ ${ }^{18} \mathrm{~F}-\mathrm{FDG}{ }^{70}$

This unique feature of the aforementioned radionuclides over the standard $2 \gamma$ radionuclides, which emit additional prompt $\gamma$ (eg, ${ }^{44} \mathrm{Sc},{ }^{66} \mathrm{Ga}$, ${ }^{82} \mathrm{Rb},{ }^{124} \mathrm{I}$ ) provides another rationale for the application of the TB-PET concept.

These radiotracers can be used in the TB-PET simultaneously and complementarily with for example, FDG to shorten the time between dosing of a radiopharmaceutical and a PET scan, which would reduce a diagnostic window, optimize the uptake and clearance of each radiotracer and minimize the risk of radiopharmaceuticals decay.

\begin{tabular}{|c|c|c|}
\hline $\begin{array}{l}\text { Cell/Tissue Molecular } \\
\text { Characteristics }\end{array}$ & Targets & Tracers (Preclinical and Clinical Studies) \\
\hline $\begin{array}{l}\text { Mutations or genome } \\
\text { instability }\end{array}$ & CAIX mutEGFR (de2-7) & $\begin{array}{l}{ }^{124} \text { I-cG250 } \\
{ }^{124} \text { I-IMP-55-R4-ch806 }\end{array}$ \\
\hline Inducing angiogenesis & $\begin{array}{l}\text { Integrin } \alpha_{\mathrm{V}} \beta_{3} \\
\text { CD105 }\end{array}$ & 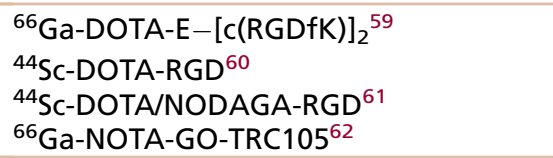 \\
\hline $\begin{array}{l}\text { Activating motility and } \\
\text { mobility }\end{array}$ & $\begin{array}{l}\text { Integrin } \alpha_{\mathrm{V}} \beta_{3} \\
\text { CD44v6 }\end{array}$ & $\begin{array}{l}{ }^{66} \mathrm{Ga}-\mathrm{DOTA}-\mathrm{E}-[\mathrm{c}(\mathrm{RGDfK})]_{2}{ }^{59} \\
{ }^{44 S c-D O T A-R G D}{ }^{60} \\
{ }^{44} \mathrm{Sc} \text {-DOTA/NODAGA-RGD } \\
{ }^{61} \\
{ }^{124} \mathrm{I}-\mathrm{cMAb} \text { U36 }\end{array}$ \\
\hline $\begin{array}{l}\text { Expressing specific surface } \\
\text { antigens }\end{array}$ & $\begin{array}{l}\text { SSTR2 } \\
\text { PSMA } \\
\text { HER2 } \\
\text { CEA }\end{array}$ & $\begin{array}{l}{ }^{44} \text { SC-DOTATOCa,64,65 } \\
{ }^{124} \text { I-J59166 } \\
{ }^{66} \mathrm{Ga} \text {-PSMA-617 } \\
\left.{ }^{449} \text { Sc-PSMA-617 }\right] \\
{ }^{124} \text { I-trastuzumab } \\
{ }^{124} \text { I-PIB-ZHER2:a } \\
{ }^{44} \text { SC-DOTA-ZHER2:342 } \\
{ }^{124} \text { I anti-CEA mAbs (cT84.66) }\end{array}$ \\
\hline
\end{tabular}

Abbreviations: CAIX, carbonic anhydrase IX, enzyme expressed in the cell membrane of clear cell renal carcinoma; CD105, endoglin, surface glycoprotein identified as angiogenesis regulator; CD44v6, type I transmembrane glycoprotein, which promotes cell adhesion and binds vascular endothelial growth factor (VEGF); CEA, Carcinoembryonic antigen; cG250, chimeric antiCAIX monoclonal antibody, girentuximab; CRGDfK, cyclo(Arg-Gly-Asp-Phe-Lys) peptide, motif specific for integrin binding; DOTA, 1,4,7,10-Tetraazacyclododecane- $\mathrm{N}, \mathrm{N}^{\prime}, \mathrm{N}^{\prime \prime}, \mathrm{N}^{\prime \prime \prime}$ - tetraacetic acid, bifunctional chelator also known as tetraxeten; DOTATATE, compound containing 3-tyrosine-octreotate and DOTA; DOTATOC, compound containing 1-phenyloalanine-3-tyrosine-octreotide and DOTA; EGFR, epidermal growth factor receptor; GO, graphen oxide; HER2, herceptin 2 receptor, receptor tyrosine-protein kinase erbB-2, also known as CD340, binding herceptin (trastuzumab); IMP-R4, MCC-Lys(MCC)-Lys(X)-d-Tyr-d-Lys(X)-OH peptide, bifunctional chelator; J591, humanized anti-PSMA monoclonal antibody; NOTA, 1,4,7-triazacyclononane-1,4,7-triacetic acid, chelator; PIB, p-iodobenzoate; PSMA, prostate-specific membrane antigen; SSTR2, somatostatin receptor type 2; TRC105, chimeric anti-CD105 monoclonal antibody; ZHER2, affibody molecule of a small (6-7 kDa) protein based on the Z domain (58 a.a.) derived from staphylococcal protein $\mathrm{A}$

${ }^{a}$ Clinical trials. 
The golden example of a dual tracer analysis application that became possible with TB-PET, is the FDA-approved ${ }^{82} \mathrm{Rb}$-Chloride, which when applied concurrently with $\left[{ }^{18} \mathrm{~F}\right]$ fludeoxyglucose (FDG) would enable simultaneous assessment of metabolic rate and perfusion, mainly in the cardiovascular system. ${ }^{54,76}$ This would allow to assess coronary arteries' occlusion and myocardium necrotic zone, as a perfusion (oxygen supply) and viability (oxygen consumption) synchronized tests.

Another example worth clinical trials, becoming possible with TB-PET, could be an early diagnostics of neuroendocrine and HER2-positive tumors via simultaneous applications of radiopharmaceuticals labeled with 2 different radionuclides, ${ }^{44} \mathrm{Sc}$ and ${ }^{18} \mathrm{~F}$, allowing for monitoring of the cancer receptor system (eg, using ${ }^{44} \mathrm{Sc}$-DOTATE) end its metabolic activity (eg, using $\left[{ }^{18} \mathrm{~F}\right] \mathrm{FDG}$ ) at the same time.

\section{Positronium Imaging}

One of the examples of multiphoton imaging is the recently proposed positronium mean lifetime tomography, ${ }^{27-30}$ which can deliver information complementary to the currently used SUV-based parameters. ${ }^{77}$ During the PET imaging, a positron emitted by the radionuclide annihilates with the electron in the patient's body, directly or via formation of the metastable positronium atom. In the human body, positronium atoms are formed in up to approximately $40 \%$ of cases of positron-electron annihilations. ${ }^{78,79}$ Positronium is an atom built from an electron and a positron (anti-electron). It is not stable but annihilates with emission of photons. In a quarter of the cases it appears as a short-lived (125 ps) para-positronium and in three-quarters of cases as ortho-positronium with mean lifetime in the vacuum of 142 ns. In the tissue ortho-positronium mean lifetime strongly depends on the size of intramolecular voids (free volumes between atoms), whereas its formation probability depends on the voids concentration (Fig. 4). In the body, the mean lifetime of ortho-positronium varies from approximately $1.8 \mathrm{~ns}$ in pure water to approximately $4 \mathrm{~ns}$ in human skin. ${ }^{80}$ Moreover, both formation probability and mean lifetime depend on the concentration of the bio-fluids and bio-active molecules ${ }^{81,82}$ and the mean lifetime can be established in the human body with the precision of approximately 20 ps. ${ }^{29}$

There are investigations demonstrating differences between normal and cancerous cells with changes of the positronium lifetime during dynamic processes undergoing in model and living biological systems. ${ }^{79-81,85-95}$ Therefore, positronium may be used as a sensor of the surrounding tissue environment, and imaging of its properties inside the patient body may serve as additional diagnostic indicator. In general, positronium imaging can be defined as a method for the position-sensitive reconstruction of positronium properties (such as mean lifetime, formation probability, and $3 \gamma / 2 \gamma$ rate ratio) within the imaged object. ${ }^{27-30}$ Imaging of positronium lifetime and its formation probability requires applications of isotopes emitting prompt gamma, while $3 \gamma / 2 \gamma$ rate ratio is accessible with all kinds of $\beta+$ emitters. $^{96}$

In case of the mean lifetime positronium image, registration of the prompt gamma is used to determine the time of the emission of the positron (which is within tens of picoseconds equal to the time of the formation of the positronium atom) and the registration of annihilation photons is used for the determination of the position and time of the positronium annihilation. ${ }^{28,29}$ The most effective way of the positronium mean lifetime image reconstruction is based on the registration of annihilations of ortho-positronium into 2 photons, which may occur in the tissue due to the pick-off $^{84}$ and conversion processes $^{82,97,98}$ and that is approximately 70 times more frequent than annihilation into 3 photons. ${ }^{28,29}$ In this case, the back-to-back photons are used to reconstruct the decay time and decay position of ortho-positronium atom on an event-by-event basis. Reconstruction of the time difference distribution between annihilation and the emission of the positron enables to determine the positronium's mean lifetime for each image voxel. ${ }^{29}$

Time resolution of positronium imaging depends predominantly on the statistics of events reconstructed in the image voxel whereas the spatial resolution depends mainly on the PET time resolution. ${ }^{29}$ Current clinical time of flight (TOF)-PETs ${ }^{12}$ achieve down to 210 ps and small laboratory type detectors reach CRT of even 30 ps equivalent to position resolution of $4.5 \mathrm{~mm}$ along the line of response. ${ }^{99}$ Therefore, with the continuous improvement of the time resolution ${ }^{25,100}$ it may become feasible in not so distant future to reconstruct positronium image directly as a density distribution of annihilation points. ${ }^{29,30}$ Resent simulation studies ${ }^{29}$ indicate that the PSF of positronium image equals to approximately $5 \mathrm{~mm}$ (radial and axial) for CRT $=50$ ps. In comparison, for the CRT of approximately 500 ps, as presently achieved by the UEXPLORER TB-PET, the PSF of positronium image would equal to $30 \mathrm{~mm}$ (radial) and $7 \mathrm{~mm}$ (axial). ${ }^{29}$ 


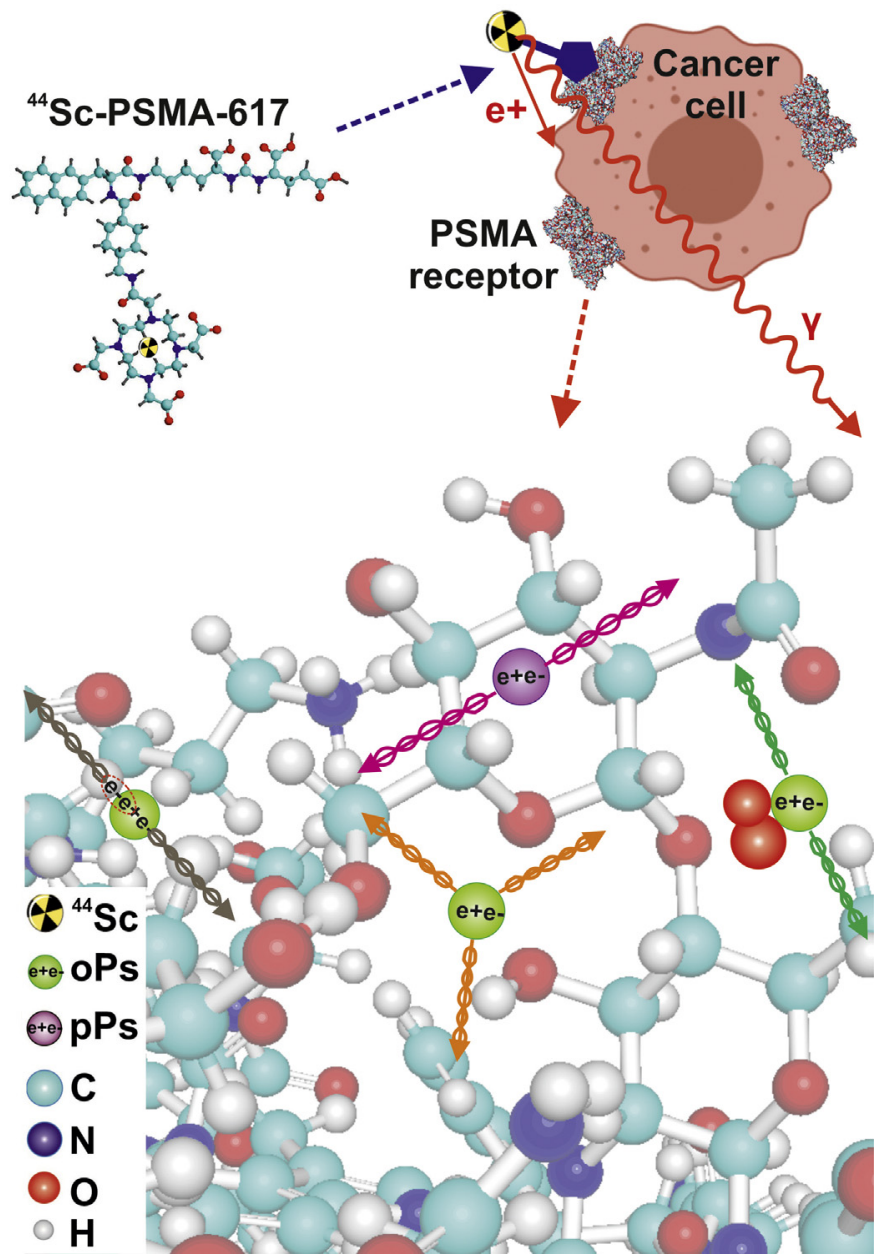

Fig. 4. Pictorial representation of the basic processes involved in the "positronium tomography" using the example of examination of the prostate cancer. The PSMA-617 ligand) labeled with radionuclide ${ }^{44} \mathrm{SC}$ attaches to the PSMA receptors highly expressed in prostate epithelial cells. ${ }^{83}$ ${ }^{44} \mathrm{Sc}$ isotope emits positron $\left(\mathrm{e}^{+}\right)$and prompt gamma $(\gamma)$ via following process: ${ }^{44} \mathrm{SC} \rightarrow{ }^{44} \mathrm{Ca}^{*} \mathrm{e}^{+} v \rightarrow{ }^{44} \mathrm{Ca} \gamma \mathrm{e}^{+}$ $v^{44}$ Positron interacting with electrons may form positronium atoms (indicated as oPs or pPs) inside cell molecules including intermolecular voids in PSMA receptors as indicated in the lower part of the sketch. Prompt gamma may be detected in the tomograph to give the signal approximately the time of positronium formation. Arrows indicate photons originating from the annihilation of para- and ortho-positronium inside free space between atoms (magenta and orange arrows), respectively. Black arrows indicate annihilation of ortho-positronium through the interaction with the electron from the surrounding molecule $e^{84}$ and green arrows illustrate photons from the conversion of ortho-into para-positronium via interaction with the oxygen molecule ${ }^{82}$ and subsequent decay of para-positronium to $2 \gamma \cdot{ }^{27}$
As an example of the possible application we use here the diagnosis of the prostate cancer. Prostate-specific membrane antigen (PSMA), (which is commonly used as a target for prostate cancer imaging and treatment) is a nonsoluble type 2 integral membrane protein with carboxypeptidase activity. ${ }^{101}$ PSMA is overexpressed in prostate cancer cells, correlates to prostatespecific antigen blood levels and reflects tumor aggressiveness diagnosed both in tissue biopsies and in prostate cancer imaging. ${ }^{102,103}$ In patients having castration-resistant prostate cancer metastasis, there is an unmet need to apply imaging-guided prostate cancer therapy based on total-body scanning and real-time theranostics. ${ }^{104}$ For this purpose [44Sc]Sc-PSMA-617 for PET imaging is the most suitable radiotracer ${ }^{68}$ (see Fig. 4). Application of [44Sc]Sc-PSMA-617 with TB-PET would enable an enhancement of accurate diagnostics by simultaneous reconstruction of the positronium image.

\section{Sensitivity of Multitracer and Positronium Imaging with Total-Body PET Scanners}

Multitracer imaging and mean lifetime positronium imaging requires registration of triple coincidences with 2 photons from $\mathrm{e}^{+} \mathrm{e}^{-}(\mathrm{Ps}) \rightarrow 2 \gamma$ annihilation and prompt gamma emitted by the $\beta^{+} \gamma$ isotope as for example, ${ }^{44} \mathrm{Sc}$. Registration of the prompt gamma enables a determination of the time of the formation of positronium, and registration of the correlated annihilation photons (2 or 3) allows for the determination of the position and time of the positronium annihilation. ${ }^{28,29,105,106}$ However, the requirement of the registration and identification of the prompt gamma, in addition to the 2 back-to-back annihilation photons, decreases significantly the imaging sensitivity with respect to the standard PET $2 \gamma$ imaging. Right panel of Fig. 3 shows sensitivity gain calculated with respect to the standard $2 \gamma$ metabolic imaging with the $20 \mathrm{~cm}$ axial-length LYSO detector 
indicated with the blue dot. The figure compares results (dashed lines) for the sensitivity gain as a function of the AFOV for multitracer and positronium imaging $(2 \gamma+$ prompt gamma) with respect to the sensitivity gain for the standard SUV imaging (solid lines). Results presented in the right panel of Fig. 3 were obtained for Scandium isotope as a $\beta^{+} \gamma$ emitter, which is decaying via the following reaction chain: ${ }^{44} \mathrm{Sc} \rightarrow{ }^{44} \mathrm{Ca}^{*} \mathrm{e}^{+} v$ $\rightarrow{ }^{44} \mathrm{Ca} \gamma \mathrm{e}^{+} v$. Scandium was chosen as an example because it is one of the most promising $\beta^{+} \gamma$ isotopes for medical applications due to (1) its convenient half-lifetime (3.9 h), (2) emission of only single prompt $1157 \mathrm{keV}$ gamma with high probability (99\%), (3) short average time of prompt gamma deexcitation (1.6 ps), (4) chemical affinity enabling labeling of for example, DTPA and DOTA-peptides which may be attached for example, to trastuzumab-herceptin or PSMA tracers, respectively, and (5) it possesses therapeutic partners $\left({ }^{47} \mathrm{Sc}\right.$ and $\left.{ }^{177} \mathrm{Lu}\right)$ emitting lowenergy electrons thus enabling theranostic approach. ${ }^{44,107-109}$

Fig. 3 indicates that for the current standard PET systems with $20 \mathrm{~cm}$ AFOV the $(2 \gamma+p r o m p t$ gamma) sensitivity is by more than order of magnitude smaller with respect to the standard $2 \gamma$ SUV imaging. However, with the increase of the AFOV the sensitivity gain of positronium and multitracer imaging increases strongly, and for the TB-PET scanner, with AFOV of $200 \mathrm{~cm}$, it exceeds the sensitivities of the standard $2 \gamma$ metabolic imaging with the PET scanners with AFOV $=20 \mathrm{~cm}$ by a factor of approximately 7.5 in case of LYSO TB-PET and by a factor of approximately 2.5 in case of the plastic TB-J-PET scanner.

\section{SUMMARY AND PROSPECTS}

The advent of TB-PET systems opens new possibilities for the personalized medicine. Increase of the AFOV to cover the total-body and the increase of whole-body scan's sensitivity by a factor of approximately 40 enables simultaneous dynamic imaging of all the organs, for the wider group of patients and diagnosis of systemic diseases. ${ }^{16}$ Recently uEXPLORER, the first TB-PET, demonstrated kinetic and parametric imaging of all tissues in the human body simultaneously. ${ }^{1,4,5}$

These new imaging capabilities deliver significant quantitative improvements in diagnostic, prognostic and theranostic assessments of various oncological, cardiological, and neurologic diseases. ${ }^{26}$ However, the extremely high cost of the UEXPLORER prevents its dissemination in hospitals and even in the research clinics. Therefore, the widespread use of TB-PET systems in a wide clinical practice will be possible only after a drastic reduction in the production cost of such tomographs.

In this article, we have opined that the reduction of crystal thickness or exchange of the LYSO by BGO crystals will not lead to a significant enough reduction of production costs, and as a solution for the economic TB-PET we described the concept of the TB-J-PET system using axially arranged long strips of plastic scintillators with readout at the edges, instead of the typical detectors built form radially arranged blocks of heavy scintillator crystals.

Although the sensitivity increase expected for the TB-PET built from plastic scintillators is by approximately a factor of 2 lower with respect to the TB-PET built from crystals, it is still significant with respect to the current $20-\mathrm{cm}$ to $25-\mathrm{cm}$-long LYSO PET systems: factor of 27 for total-body imaging and factor of approximately 2 for single organ imaging. Yet, the mechanical robustness of plastics with respect to crystals enable to make the plastic total-body scanner lightweight, modular and portable. A photograph of the 24module prototype of such a scanner with $50 \mathrm{~cm}$ AFOV is shown in the right panel of Fig. 2. Modularity enables construction of PET with imaging chamber adjustable to the size of the patient, which could be of advantage in case of imaging of for example, obese or claustrophobic patients. Plastic TB-PET is a cost-effective solution for the long axial FOV PET scanner. Total cost of components of the plastic TB-PET is approximately 5 times less with respect to the crystal-based TBPET systems. Cost of crystal scintillators constitutes approximately $50 \%$ of the total costs of the TB-PET scanner. ${ }^{16}$ Plastic scintillators are more than an order of magnitude less expensive than LYSO crystals. Electronics and SiPMs constitute another $\sim 50 \%$ of the costs of the crystal TBPET, and in case of plastic PET with axially arranged scintillator strips these costs are significantly reduced because the readout is placed mainly at the ends of the cylindrical detector compared with the coverage of the full cylinder surface in case of the crystal PET detectors. Thus in case of plastic TB-PET, the cost of electronics are proportional to the trans-axial crosssection of the detector, whereas in case of crystal TB-PET they are proportional to the area of the detection cylinder. Overall the plastic TB-J-PET may be more than factor of 5 less expensive than the crystal-based TB-PET, making it a realistic cost-effective solution for the broad clinical applications.

High sensitivity and long AFOV of TB-PET opens new possibilities for diagnosis beyond the static 
SUV index, improving diagnostic assessment specificity by access to kinetic and parametric imaging of all organs of the patient, simultaneously. ${ }^{13,14}$ Then we presented examples of further diagnostic benefits of TB-PET system that arise from the possibility of effective multiphoton imaging opening prospects for the multitracer and positronium imaging and thus giving perspectives for the further quantitative increase of the diagnosis specificity. In the current PET imaging the prompt gamma emitted by $\beta^{+} \gamma$ isotopes as for example, ${ }^{44} \mathrm{Sc}$ constitutes a source of background. However, high sensitivity, triggerless data acquisition ${ }^{36}$ and dedicated data selection algorithms ${ }^{28,29,37,105,106,110,111}$ available at J-PET enable efficient registration of triple coincidences (see Fig. 3) corresponding to events with 2 backto-back annihilation photons and prompt gamma. The additional information carried out by prompt gamma may be used for (1) disentanglement of images from different radiopharmaceuticals in case of simultaneous multitracer imaging, ${ }^{48}$ and for (2) determination of the positronium mean lifetime image. $^{29}$

Assessing of tumor status using multiple PET tracers has great potential for personalized oncology. ${ }^{112}$ In clinical practice multitracer imaging may decrease significantly the time needed for the sequential imaging with more than 1 radiopharmaceutical that currently needs to be long between subsequent scans because of the long (many hours) biological decay time needed for cleaning up a tracer from the organism. Imaging with 2 different tracers at once is currently impractical with standard $2 \gamma$ PET though it could enhance significantly the diagnostic possibilities (for example, by an early diagnostics of neuroendocrine and HER2 positive tumors) by delivering information of the tumor location (eg, from ${ }^{18} \mathrm{~F}$ labeled radiopharmaceuticals) and simultaneously independent information of the tumor type (cancer receptor system) by use of for example, ${ }^{44} \mathrm{Sc}$ DOTATATE radiopharmaceuticals, where DOTATATE possesses high binding affinity for the somatostatin receptors enhancing diagnostics quality of neuroendocrine cancers. ${ }^{64}$ The scope of applications of radiopharmaceuticals labeled with Scandium $\left({ }^{43} \mathrm{Sc},{ }^{44} \mathrm{Sc}\right)$ is growing, particularly for oncologic imaging purposes. ${ }^{64,113-116}$ Clinical trials with ${ }^{44} \mathrm{Sc}$ may be enhanced by the application of TB-J-PET which in addition to SUV and kinetic parametric images would deliver positronium lifetime image and may enable simultaneous double-tracer imaging. Finally it is worth stressing that a golden example of dual tracer analysis, becoming possible with TB-J-PET, could be a concurrent application of FDA-approved
${ }^{82} \mathrm{Rb}$-Chloride and $\left[{ }^{18} \mathrm{~F}\right] \mathrm{FDG}$, allowing simultaneous assessment of myocardium metabolic rate and perfusion of the cardiovascular system.

\section{ACKNOWLEDGMENTS}

Authors are thankful to Prof. Stan Majewski and Dr Aleksander Gajos for reading and correcting the article, to Prof. Steven Bass and Prof. Jan Stanek for the perusal of the article and useful comments, and to Jyoti Chhokar, Dr Agnieszka Kamińska, Dr Daria Kisielewska, Pawel Kowalski, Szymon Parzych, and Shivani and Monika Szczepanek for the help in the preparation of the figures and calculations. The authors acknowledge support by the Foundation for Polish Science through the TEAM PIOR.04.04.00-00-4204/17 programme and by the Polish Ministry for Science and Higher Education through grant no. 7150/E-338/SPUB/2017/1.

\section{REFERENCES}

1. Badawi RD, Shi H, Hu P, et al. First human imaging studies with the EXPLORER total-body PET scanner. J Nucl Med 2019;60(3):299-303.

2. Karp JS, Viswanath V, Geagan MJ, et al. PennPET explorer: design and preliminary performance of a whole-body imager. J Nucl Med 2020;61(1): 136-43.

3. Cherry SR, Badawi RD, Karp JS, et al. Total-body imaging: transforming the role of positron emission tomography. Sci Transl Med 2017;9(381):eaaf6169.

4. Cherry SR, Jones T, Karp JS, et al. Total-body PET: maximizing sensitivity to create new opportunities for clinical research and patient care. J Nucl Med 2018;59(1):3-12.

5. Zhang X, Xie Z, Berg E, et al. Total-body dynamic reconstruction and parametric imaging on the UEXPLORER. J Nucl Med 2020;61(2):285-91.

6. Surti S, Viswanath V, Daube-Witherspoom ME, et al. Benefit of improved performance with stateof-the art digital PET/CT for lesion detection in oncology. J Nucl Med 2020;120:242305.

7. Schmall JP, Karp JS, Alavi A. The potential role of total body PET imaging in assessment of atherosclerosis. PET Clin 2019;14(2):245-50.

8. McKenney-Drake ML, Moghbel MC, Paydary K et al. 18F-NaF and 18F-FDG as molecular probes in the evaluation of atherosclerosis. Eur $\mathrm{J} \mathrm{Nucl}$ Med Mol Imaging 2018;45(12):2190-200.

9. Nakajima R, Abe K, Sakai S. IgG4-related diseases; whole-body FDG-PET/CT may be easier to evaluate rare lesions. J Nucl Med 2017;58(suppl. 1):943.

10. Yamashita $H$, Kubota K, Mimori A. Clinical value of whole-body PET/CT in patients with active rheumatic diseases. Arthritis Res Ther 2014;16(5):423. 
11. Grant AM, Deller TW, Khalighi MM, et al. NEMA NU 2-2012 performance studies for the SiPM-based ToF-PET component of the GE SIGNA PET/MR system. J Med Phys 2016;43(5):2334.

12. van Sluis J, de Jong J, Schaar J, et al. Performance characteristics of the digital biograph vision PET/ CT system. J Nucl Med 2019;60(7):1031-6.

13. Zhang X, Xie Z, Berg E, et al. Total-body parametric imaging using kernel and direct reconstruction on the UEXPLORER. J Nucl Med 2019;60(suppl. 1):456.

14. Deng Z, Hu D, Ding Y, et al. A comparison of image quality with $\mathrm{UMI} 780$ and the first total-body uEXPLORER scanner. J Nucl Med 2019;60(suppl. 1):381.

15. Houshmand S, Salavati A, Hess S, et al. An update on novel quantitative techniques in the context of evolving whole-body PET imaging. PET Clin 2015; 10(1):45-58.

16. Vandenberghe S, Moskal P, Karp J. State of the art in total body PET. EJNMMI Phys 2020;7(1):35.

17. Surti S, Werner M, Karp J. Study of PET scanner designs using clinical metrics to optimize the scanner axial FOV and crystal thickness. Phys Med Biol 2013;58(12):3995-4012.

18. Zhang J, Knopp MI, Knopp MV. Sparse detector configuration in SiPM digital photon counting PET: a feasibility study. Mol Imaging Biol 2019;21(3): 447-53.

19. Zein SA, Karakatsanis NA, Issa M, et al. Physical performance of a long axial field-of-view PET scanner prototype with sparse rings configuration: a Monte Carlo simulation study. Med Phys 2020; 47(4):1949-57.

20. Zhang $Y$, Wong $W H$. System design studies for a low-cost high-resolution BGO PET with 1-meter axial field of view. J Nucl Med 2017;58(suppl.1):221.

21. Gonzalez-Montoro A, Sanchez F, Majewski S, et al. Highly improved operation of monolithic BGO-PET blocks. J Instrum 2017;12:C11027.

22. Brunner S, Schaart D. BGO as a hybrid scintillator/ Cherenkov radiator for cost-effective time-of-flight PET. Phys Med Biol 2017;62(11):4421-39.

23. Kwon SI, Roncali E, Gola A, et al. Dual-ended readout of bismuth germanate to improve timing resolution in time-of-flight PET. Phys Med Biol 2019;64(10):105007.

24. Cates JW, Levin CS. Electronics method to advance the coincidence time resolution with bismuth germanate. Phys Med Biol 2019;64(17): 175016.

25. Gundacker S, Martinez Turtos R, Kratochwil N, et al. Experimental time resolution limits of modern SiPMs and TOF-PET detectors exploring different scintillators and Cherenkov emission. Phys Med Biol 2020;65(2):025001.

26. Majewski S. Imaging is Believing: The Future of Human Total Body Molecular Imaging Starts Now IL
NUOVO CIMENTO 43 C (2020) 8, 3-5 September 2019, Accademia degli Zelanti e dei Dafnici, Acireale, Catania, Italy Proceedings of the FATA2019: FAst Timing Applications for nuclear physics and medical imaging.

27. Moskal P, Jasińska B, Stępień EŁ, et al. Positronium in medicine and biology. Nat Rev Phys 2019;1: 527-9.

28. Moskal P, Kisielewska D, Curceanu C, et al. Feasibility study of the positronium imaging with the J-PET tomograph. Phys Med Biol 2019;64(5): 055017.

29. Moskal P, Kisielewska D, Shopa R, et al. Performance assessment of the $2 \gamma$ positronium imaging with the total-body PET scanners. EJNMMI Phys 2020; 7(1):44.

30. Moskal P. Positronium Imaging. 2019 IEEE Nuclear Science Symposium and Medical Imaging Conference, Convention Centre, Manchester, UK, 26 October-2nd of November, 2019 (NSS/MIC) https:// doi.org/10.1109/NSS/MIC42101.2019.9059856.

31. Moskal P, Niedźwiecki Sz, Bednarski T, et al. Test of a single module of the J-PET scanner based on plastic scintillators. Nucl Instrum Methods Phys Res A 2014;764:317-21.

32. Saint Gobain. Available at: https://www.crystals. saint-gobain.com/. Accessed date: April 25, 2020.

33. Vilardi I, Braem A, Chesi E, et al. Optimization of the effective light attenuation length of YAP:Ce and LYSO:Ce crystals for a novel geometrical PET concept. Nucl Instrum Methods Phys Res A 2006;564:506-14.

34. Mao R, Zhang L, Zhu Y R. Optical and scintillation properties of inorganic scintillators in high energy physics. IEEE Trans Nucl Sci 2008;55(4):2425-31.

35. Pałka M, Strzempek P, Korcyl G, et al. Multichannel FPGA based MVT system for high precision time (20 ps RMS) and charge measurement. J Instrum 2017; 12:P08001.

36. Korcyl G, Białas P, Curceanu C, et al. Evaluation of single-chip, real-time tomographic data processing on FPGA SoC devices. IEEE Trans Med Imaging 2018;37(11):2526-35.

37. Krzemień W, Gajos A, Gruntowski A, et al. Analysis framework for the J-PET scanner. Acta Phys Pol A 2015;127:1491-4.

38. Raczyński L, Moskal P, Kowalski P, et al. Compressive sensing of signals generated in plastic scintillators in a novel J-PET instrument. Nucl Instrum Methods Phys Res A 2015;786:105-12.

39. Moskal P, Zoń N, Bednarski T, et al. A novel method for the line-of-response and time-of-flight reconstruction in TOF-PET detectors based on a library of synchronized model signals. Nucl Instrum Methods Phys Res A 2015;775:54-62.

40. Raczyński L, Moskal P, Kowalski P, et al. Novel method for hit-positon reconstruction using voltage 
signals in plastic scintillators and its application to the Positron Emission Tomography. Nucl Instrum Methods Phys Res A 2014;764:186-92.

41. Eljen technology, Physical Constants of plastic scintillators. Available at: https://eljentechnology. com/images/technical_library/Physical_Constants_ Plastic.pdf. Accessed date: April 25, 2020.

42. Mao R, Chen Wu C, Dai LE, et al. Crystal growth and scintillation properties of LSO and LYSO crystals. J Cryst Growth 2013;368:97-100.

43. Moskal P, Rundel O, Alfs D, et al. Time resolution of the plastic scintillator strips with matrix photomultiplier readout for J-PET tomograph. Phys Med Biol 2016;61(5):2025-47.

44. Sitarz M, Cussonneau JP, Matulewicz T, et al. Radionuclide candidates for $\beta+\gamma$ coincidence PET: an overview. Appl Radiat Isot 2020;155: 108898.

45. Kowalski K, Wiślicki W, Shopa RY, et al. Estimating the NEMA characteristics of the J-PET tomograph using the GATE package. Phys Med Biol 2018; 63(16): 165008.

46. Jan S, Benoit D, Becheva E, et al. GATE v6: a major enhancement of the GATE simulation platform enabling modelling of CT and radiotherapy. Phys Med Biol 2011;56(4):881-901.

47. Smyrski J, Alfs D, Bednarski T, et al. Measurement of gamma quantum interaction point in plastic scintillator with WLS strips. Nucl Instrum Methods Phys Res A 2017;851:39-42.

48. Gajos A, Kamińska D, Moskal P, et al. Method for reconstructing multi-tracer metabolic and morphometric images and tomography system for multi-tracer metabolic and morphometric imaging. Patent Number: US 10339676. Official Gazette of the United States Patent and Trademark Office Patents; 2019.

49. Grignon C, Barbet J, Bardie M, et al. Nuclear medical imaging using $\beta+\gamma$ coincidences from 44Sc radio-nuclide with liquid Xenon as detection medium. Nucl Instrum Methods Phys Res A 2007; 571:42-5.

50. Donnard J, Chen W-T, Cussonneau J-P, et al. Compton imaging with liquid Xenon and 44Sc: recent progress toward 3 gamma imaging. Nucl Med Rev 2012;15(supp.C):64-7.

51. Lang C, Habs D, Parodi K, et al. Sub-millimeter nuclear medical imaging with high sensitivity in positron emission tomography using $\beta+\gamma$ coincidences. J Instrum 2014;9:P01008.

52. Oger T, Chen W-T, Cussonneau J-P, et al. A liquid xenon TPC for a medical imaging Compton telescope. Nucl Instrum Methods Phys Res A 2012; 695:125-8.

53. Thirolf PG, Lang C, Parodi K. Perspectives for highlysensitive PET-based medical imaging using $\beta+\gamma$ coincidences. Acta Phys Pol A 2015;127:1441-4.
54. U.S. Food and Drug Administration. Available at: https://www.fda.gov/home https://www.cardinal health.com/content/dam/corp/web/documents/factsheet/cardinal-health-fda-approved-radiopharma ceuticals.pdf. Accessed date: April 25, 2020.

55. Cheal SM, Punzalan B, Doran MG, et al. Pairwise comparison of 89Zr- and 124l-labeled cG250 based on positron emission tomography imaging and nonlinear immunokinetic modeling: in vivo carbonic anhydrase IX receptor binding and internalization in mouse xenografts of clear-cell renal cell carcinoma. Eur J Nucl Med Mol Imaging 2014; 41(5):985-94.

56. Larson SM, Motzer RJ, Pandit-Taskar N, et al. 124IcG250 PET scan for early detection of response to sunitinib in patients (Pts) with metastatic clear cell renal cell carcinoma (MccRCC). J Clin Oncol 2012;30(0):362.

57. Khandani A, Wallen E, Rathmell K, et al. 124lcG250 PET in clear cell renal cell carcinoma (ccRCC). J Nucl Med 2014;55(supp.1):1348.

58. Lee FT, O'Keefe GJ, Gan HK, et al. Immuno-PET quantitation of de2-7 epidermal growth factor receptor expression in glioma using 124I-IMP-R4labeled antibody ch806. J Nucl Med 2010;51: 967-72.

59. Lopez-Rodriguez V, Gaspar-Carcamo RE, Pedraza-Lopez M, et al. Preparation and preclinical evaluation of (66)Ga-DOTA-E(c(RGDfK))2 as a potential theranostic radiopharmaceutical. Nucl Med Biol 2015;42(2):109-14.

60. Hernandez R, Valdovinos $H$, Chakravarty $R$, et al. 44Sc-labeled cyclic RGD peptide for PET imaging of integrin $\alpha$ Vß3. J Nucl Med 2014;55(supp.1):6.

61. Domnanich KA, Müller C, Farkas R, et al. 44Sc for labeling of DOTA- and NODAGA-functionalized peptides: preclinical in vitro and in vivo investigations. EJNMMI Radiopharm Chem 2017;1(1):8 [published correction: 2018;3:13].

62. Hong $\mathrm{H}$, Zhang $\mathrm{Y}$, Engle JW, et al. In vivo targeting and positron emission tomography imaging of tumor vasculature with 66Ga-labeled nano-graphene. Biomaterials 2012;339160:4147-56.

63. Fortin MA, Salnikov AV, Nestor M, et al. ImmunoPET of undifferentiated thyroid carcinoma with radioiodine-labelled antibody cMAb U36: application to antibody tumour uptake studies. Eur J Nucl Med Mol Imaging 2007;34(9):1376-87.

64. Singh A, van der Meulen NP, Müller C, et al. First-inhuman PET/CT imaging of metastatic neuroendocrine neoplasms with cyclotron-produced 44Sc-DOTATOC: a proof-of-concept study. Cancer Biother Radiopharm 2017;32(4):124-32.

65. Rösch F, Baum RP. Generator-based PET radiopharmaceuticals for molecular imaging of tumours: on the way to theranostics. Dalton Trans 2011; 40(23):6104-11. 
66. Fung EK, Cheal SM, Fareedy SB, et al. Targeting of radiolabeled $\mathrm{J} 591$ antibody to PSMA-expressing tumors: optimization of imaging and therapy based on non-linear compartmental modeling. EJNMMI Res 2016;6(1):7.

67. Amor-Coarasa A, Kelly JM, Ponnala S, et al. 66Ga: a Novelty or a valuable preclinical screening tool for the design of targeted radiopharmaceuticals? Molecules 2018;23(10):2575.

68. Eppard E, de la Fuente A, Benešová M, et al. Clinical translation and first in-human use of [44Sc]ScPSMA-617 for PET imaging of metastasized castrate-resistant prostate cancer. Theranostics 2017;7(18):4359-69.

69. Khawar A, Eppard E, Sinnes JP, et al. [44Sc]ScPSMA-617 Biodistribution and dosimetry in patients with metastatic castration-resistant prostate carcinoma. Clin Nucl Med 2018;43(5):323-30.

70. Guo X, Zhou N, Chen Z, et al. Construction of 124ltrastuzumab for noninvasive PET imaging of HER2 expression: from patient-derived xenograft models to gastric cancer patients. Gastric Cancer 2020. https://doi.org/10.1007/s10120-019-01035-6.

71. Orlova $A$, Wållberg $H$, Stone-Elander $S$, et al. On the selection of a tracer for PET imaging of HER2-expressing tumors: direct comparison of a 124l-labeled affibody molecule and trastuzumab in a murine xenograft model. J Nucl Med 2009; 50930:417-25.

72. Honarvar H, Müller C, Cohrs S, et al. Evaluation of the first 44Sc-labeled Affibody molecule for imaging of HER2-expressing tumors. Nucl Med Biol 2017;45:15-21.

73. Bading JR, Hörling M, Williams LE, et al. Quantitative serial imaging of an 124I anti-CEA monoclonal antibody in tumor-bearing mice. Cancer Biother Radiopharm 2008;23(4):399-409.

74. Ruigrok EAM, van Weerden WM, Nonnekens J, et al. The future of PSMA-targeted radionuclide therapy: an overview of recent preclinical research. Pharmaceutics 2019;11(11):560.

75. Sihver W, Pietzsch J, Krause M, et al. Radiolabeled cetuximab conjugates for EGFR targeted cancer diagnostics and therapy. Pharmaceuticals (Basel) 2014; 7(3):311-38.

76. Dibble EH, Yoo DC. Precision medicine and PET/ Computed tomography in cardiovascular Disorders. PET Clin 2017;12:459-73.

77. Conti M, Bendriem B. The new opportunities for high time resolution clinical TOF PET. Clin Transl Imaging 2019;7:139-47.

78. Harpen MD. Positronium: review of symmetry, conserved quantities and decay for the radiological physicist. Med Phys 2004;31(1):57-61.

79. Jasińska B, Zgardzińska B, Chołubek G, et al. Human tissues investigation using PALS Technique. Acta Phys Pol B 2017;48:1737-47.
80. Chen H, Van Horn J, Ching Jean Y. Applications of positron annihilation spectroscopy to life science. Defect and Diffusion Forum 2012;331: 275-93.

81. Jasińska B, Zgardzińska B, Chołubek G, et al. Human tissue investigations using PALS technique free radicals influence. Acta Phys Pol A 2017;132: 1556-9.

82. Stepanov PV, Selim FA, Stepanov SV, et al. Interaction of positronium with dissolved oxygen in liquids. Phys Chem Chem Phys 2020;22:5123-31.

83. Rahbar K, Afshar-Oromieh A, Jadvar $H$, et al. PSMA theranostics: current status and future directions. Mol Imaging 2018;17:1-9.

84. Garwin RL. Thermalization of positrons in metals. Phys Rev 1953;91:1571-2.

85. Kilburn D, Townrow S, Meunier V, et al. Organization and mobility of water in amorphous and crystalline trehalose. Nat Mater 2006;5:632-5.

86. Jean YC, Li Y, Liu G, et al. Applications of slow positrons to cancer research: search for selectivity of positron annihilation to skin cancer. Appl Surf Sci 2006;252:3166-71.

87. Jean $Y$, Chen H, Liu G, et al. Life science research using positron annihilation spectroscopy: UVirradiated mouse skin Radiat. Phys Chem 2007; 76:70-5.

88. Liu G, Chen H, Chakka L, et al. Applications of positron annihilation to dermatology and skin cancer. Phys Status Solidi C 2007;4:3912-5.

89. Liu G, Chen H, Chakka L, et al. Further search for selectivity of positron annihilation in the skin and cancerous systems. Appl Surf Sci 2008;255: 115-8.

90. Yas RM, Al-Mshhdani AH, Elias MM, et al. Detection of line shape parameters in normal and abnormal biological tissues. Iraqi J Phys 2012;10: 77-82

91. Axpe E, Lopez-Euba T, Castellanos-Rubio A, et al. Detection of atomic scale changes in the free volume void size of three-dimensional colorectal cancer cell culture using positron annihilation lifetime spectroscopy. PLoS One 2014;9:1-5.

92. Pietrzak R, Borbulak S, Szatanik R. Influence of neoplastic therapy on the investigated blood using positron annihilation lifetime spectroscopy. Nukleonika 2013;58:199-202.

93. Kubicz E, Jasińska B, Zgardzińska B, et al. Studies of unicellular micro-organisms Saccharomyces cerevisiae by means of positron annihilation lifetime spectroscopy. Nukleonika 2015;60:749-53.

94. Kubicz E. Potential for biomedical applications of positron annihilation lifetime spectroscopy (PALS). AIP Conf Proc 2019;2182:050004.

95. Bura Z, Dulski K, Kubicz E, et al. Studies of the ortho-Positronium lifetime for cancer diagnostic. Acta Phys Pol B 2020;51:377. 
96. Jasińska B, Moskal P. A new PET diagnostic indicator based on the ratio of $3 \gamma / 2 \gamma$ positron annihilation. Acta Phys Pol B 2017;48:1577.

97. Consolati G, Quasso F. Positronium-oxygen interactions in polytrimethylsilylpropine membranes. Appl Phys 1998;B66:371-6.

98. Zgardzińska B, Białko W, Jasińśka B. Ortho-para spin conversion of Ps by paramagnetic $\mathrm{O} 2$ dissolved inorganic compounds. Nukleonika 2015; 60(4):801-4.

99. Ota R, Nakajima K, Ogawa I, et al. Coincidence time resolution of 30 ps FWHM using a pair of Cherenkov-radiator integrated MCPPMTs. Phys Med Biol 2019;64(7):07LT01.

100. Lecoq P. Pushing the limits in time-of-flight pet imaging. IEEE Trans Rad Plasma Med Sci 2017;1: 473-85.

101. Silver DA, Pellicer I, Fair WR, et al. Prostate-specific membrane antigen expression in normal and malignant human tissues. Clin Cancer Res 1997; 3(1):81-5.

102. Bravaccini S, Puccetti M, Bocchini M, et al. PSMA expression: a potential ally for the pathologist in prostate cancer diagnosis. Sci Rep 2018;8(1): 4254.

103. Pereira Mestre R, Treglia G, Ferrari M, et al. Correlation between PSA kinetics and PSMA-PET in prostate cancer restaging: a meta-analysis. Eur J Clin Invest 2019;49(3):e13063.

104. Michalski K, Mix M, Meyer PT, et al. Determination of whole-body tumour burden on [68Ga]PSMA-11 PET/CT for response assessment of [177Lu] PSMA-617 radioligand therapy: a retrospective analysis of serum PSA level and imaging derived parameters before and after two cycles of therapy. Nuklearmedizin 2019;58(6):443-50.

105. Gajos A, Kamińska D, Czerwiński E, et al. Trilateration-based reconstruction of ortho-positronium decays into three photons with the J-PET detector. Nucl Instrum Methods Phys Res A 2016;819:54-9.

106. Kamińska D, Gajos A, Czerwiński E, et al. A feasibility study of ortho-positronium decays measurement with the J-PET scanner based on plastic scintillators. Eur Phys J C Part Fields 2016;76:445.

107. Müller C, Bunka M, Haller S, et al. Promising prospects for 44Sc-/47Sc-based theragnostics: application of $47 \mathrm{Sc}$ for radionuclide tumor therapy in mice. J Nucl Med 2014;55(10):1658-64.

108. Umbricht CA, Benesova M, Schmid RM, et al. 44Sc-PSMA-617 for radiotheragnostics in tandem with 177Lu-PSMA-617-preclinical investigations in comparison with 68Ga-PSMA-11 and 68GaPSMA-617. EJNMMI Res 2017;7(1):9.

109. Müller C, Domnanich KA, Umbricht CA, et al. Scandium and terbium radionuclides for radiotheranostics: current state of development towards clinical application. Br J Radiol 2018;91:20180074.

110. Dulski K, Curceanu C, Czerwiński E, et al. Commissioning of the J-PET detector in view of the positron annihilation lifetime spectroscopy. Hyperfine Interact 2018;239:40.

111. Dulski K, Zgardzińska B, Białas P, et al. Analysis procedure of the positronium lifetime spectra for the J-PET detector. Acta Phys Pol A 2017;132: 1637-40.

112. Kadrmas DJ, Hoffman JM. Methodology for quantitative rapid multi-tracer PET tumor characterizations. Theranostics 2013;3(10):757-73.

113. Hofman MS, Kong G, Neels OC, et al. High management impact of Ga-68 dotatate (gatate) PET/ CT for imaging neuroendocrine and other somatostatin expressing tumours. J Med Imaging Radiat Oncol 2012;56(1):40-7.

114. Krajewski S, Cydzik I, Abbas K, et al. Cyclotron production of 44Sc for clinical application. Radiochim Acta 2013;101:333-8.

115. Huclier-Markai S, Kerdjoudj R, Alliot C, et al. Optimization of reaction conditions for the radiolabeling of DOTA and DOTA-peptide with $44 \mathrm{~m} / 44 \mathrm{Sc}$ and experimental evidence of the feasibility of an in vivo PET generator. Nucl Med Biol 2014; 41(Suppl):e36-43.

116. Walczak R, Krajewski S, Szkliniarz K, et al. Cyclotron production of $43 \mathrm{Sc}$ for PET imaging. EJNMMI Phys 2015;2:33. 